
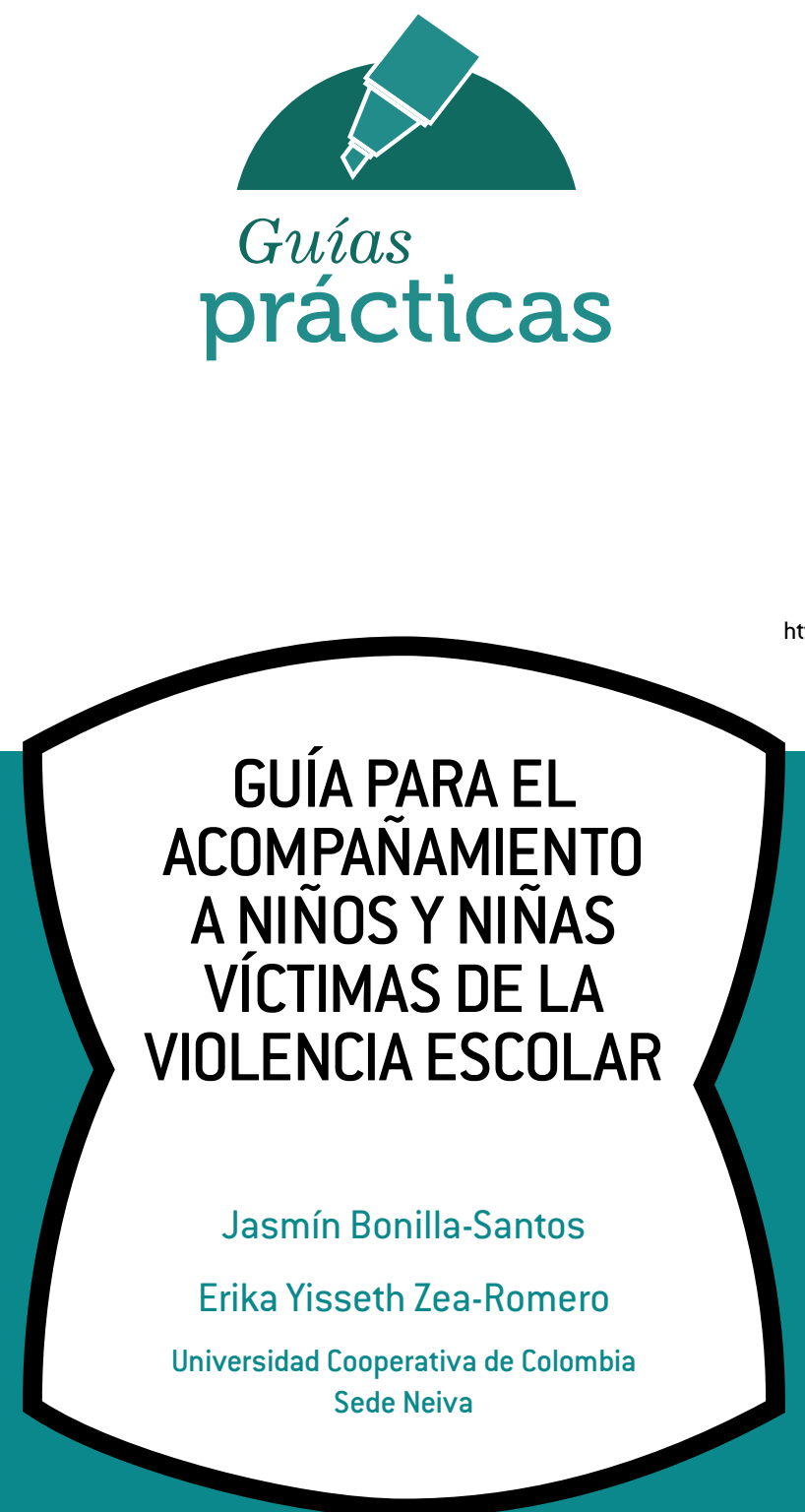


\section{ACERCA DE LAS AUTORAS}

Jasmín Bonilla-Santos. Ph. D. en Psicología

con énfasis en Neurociencia; Coordinadora de Investigación, Universidad Cooperativa de

Colombia, sede Neiva.

Correo electrónico:

jasmin.bonillas@campusucc.edu.co

ORCID: https://orcid.org/0000-0001-7660-2209

Erika Yisseth Zea-Romero. Psicóloga, joven investigadora Minciencias, Universidad Cooperativa de Colombia, sede Neiva.

Correo electrónico: erik_30.01@hotmail.com

ORCID: https://orcid.org/0000-0003-0695-3411

\section{CÓMO CITAR ESTE DOCUMENTO}

Bonilla Santos, J. y Zea Romero, E. Y. (2021). Guía para el acompañamiento a niños y niñas víctimas de la violencia escolar (Generación de contenidos impresos N.26). Ediciones Universidad Cooperativa de Colombia. https://doi.org/10.16925/gcgp.40

NOTA LEGAL

El presente documento de trabajo ha sido incluido dentro de nuestro repositorio institucional como Apropiación social de conocimiento por solicitud del autor, con fines informativos, educativos o académicos. Asimismo, los argumentos, datos y análisis incluidos en el texto son responsabilidad absoluta del autor y no representan la opinión del Fondo Editorial o de la Universidad.

\section{DISCLAIMER}

This coursework paper has been uploaded to our institutional repository as Social Appropriation of Knowledge due to the request of the author. This document should be used for informational, educational or academic purposes only. Arguments, data and analysis included in this document represent authors' opinion not the Press or the University.

(c) (1) () $)$ Este documento puede ser consultado, descargado o reproducido desde nuestro repositorio institucional (http://repository. ucc.edu.co/handle/20.500.12494/7369) para uso de sus contenidos, bajo la licencia de Creative Commons Reconocimiento-NoComercial-SinObraDerivada 4.0 Internacional. http://creativecommons.org/licenses/by-nc-nd/4.0/ 


\section{TABLA DE CONTENIDO}

Conceptualización de la violencia escolar 5

Causas posibles de la violencia escolar 5

Programas educativos contra la violencia escolar 5

Acoso escolar 6

Tipos de acoso escolar 6

Características de los actores del acoso escolar $\quad 7$

Consecuencias del acoso escolar $\quad 7$

Factores de riesgo del acoso escolar $\quad 8$

Intervención en el acoso escolar 9

Neuropsicología del acoso escolar 9

$\begin{array}{ll}\text { Cognición social } & 10\end{array}$

$\begin{array}{ll}\text { Actividades } & 11\end{array}$

$\begin{array}{ll}\text { Referencias } & 16\end{array}$ 


\title{
26 GUÍA PARA EL ACOMPAÑAMIENTO A NIÑOS Y NIÑAS VÍCTIMAS DE LA VIOLENCIA ESCOLAR
}

\author{
Jasmín Bonilla-Santos \\ Erika Yisseth Zea-Romero
}

\section{Resumen}

El propósito de esta guía es abordar una temática considerada como un problema en el ámbito mundial en los diferentes contextos educativos, como lo es la violencia escolar, definida como cualquier tipo de violencia que se da en contextos escolares y puede ir dirigida hacia alumnos, profesores o propiedades. Sin embargo, es importante destacar que esta guía se centrará específicamente en un tipo de violencia que afecta la salud mental de niños y adolescentes: el acoso escolar, considerada como cualquier forma de maltrato físico, psicológico y verbal producido entre estudiantes de manera reiterada a lo largo de un determinado tiempo, tanto en las aulas como en los diferentes contextos. Asimismo, busca identificar las características, consecuencias, causas posibles de la violencia escolar y programas educativos que previenen el maltrato entre pares y promueven espacios de comunicación asertiva y buenas relaciones interpersonales.

Palabras clave: acoso escolar, educación, intervención, violencia escolar. 


\section{CONCEPTUALIZACIÓN DE LA VIOLENCIA ESCOLAR}

La violencia escolar es una de las principales problemáticas evidenciadas en los contextos educativos debido a la frecuencia con que se presenta y a las consecuencias que deriva a nivel emocional, social y personal de niños y adolescentes (Ayala-Carrillo, 2015). Es definida como aquella acción u omisión dañina que se realiza de forma intencionada por parte de los miembros de la comunidad educativa (alumnos, profesores, padres, entre otros). Los espacios donde se llevan a cabo estas acciones son principalmente en las instalaciones educativas, pero también en los alrededores de las escuelas o en lugares donde se desarrollan actividades extraescolares.

La violencia escolar es consecuencia de la participación de los individuos que integran la escuela y ejercen conductas violentas aprendidas desde su contexto social y desarrolladas en el ámbito educativo. Es considerada como el uso inadecuado de poder que ejerce cualquier miembro de la comunidad educativa, afectando la dinámica escolar, provocando daños a personas, vulnerando los derechos de las víctimas y atentando contra el desarrollo de la personalidad de los involucrados (Rodríguez et al., 2020).

La mayoría de las investigaciones sobre violencia escolar se centran principalmente en el fenómeno del bullying, aunque también existen otros tipos de violencia escolar como la violencia interpersonal, la violencia de género o la violencia por parte de los alumnos hacia los docentes y entre los mismos docentes (Ayala-Carrillo, 2015).

\section{CAUSAS POSIBLES DE LA VIOLENCIA ESCOLAR}

Entre los factores que explican la violencia en la escuela se señala que el contexto familiar es fundamental, debido a las problemáticas que se evidencian como consecuencia de la ausencia de pautas de crianza por parte de los progenitores, falta de cariño, escasa comunicación, pobreza, privaciones, entre otras (Martínez, 2010). De igual manera, el contexto escolar es considerado como un eje fundamental de convivencia; sin embargo, algunos comportamientos como el hostigamiento, el ausentismo recurrente de maestros, la improvisación de clases, la falta de control del profesor, la indisciplina, el racismo y la intolerancia impiden un buen ambiente académico y educativo. Asimismo, cabe mencionar que los medios de comunicación han generado en los individuos conductas inadecuadas que conllevan ambientes hostiles y de violencia representados a través de videojuegos, programas de televisión, noticias y películas (Prieto, 2005).

Es importante resaltar que las conductas agresivas las llevan a cabo con mayor frecuencia estudiantes que se sienten integrados y aceptados por un grupo; entre más agresivo es este, los ataques hacia sus compañeros son más violentos (Prieto, 2005).

No obstante, aunque las instituciones educativas son consideradas como un espacio de construcción de identidades, lo que sucede en su interior es un reflejo de lo que sucede afuera, en los diferentes contextos familiares y sociales. Por lo tanto, las interrelaciones que se dan entre los individuos se desarrollan de acuerdo con las experiencias previas de cada uno con el mundo exterior (Ayala-carrillo, 2015).

\section{PROGRAMAS EDUCATIVOS CONTRA LA VIOLENCIA ESCOLAR}

Los programas de intervención enfocados en la violencia escolar están diseñados, en su mayoría, para prevenir y disminuir conductas violentas a través de intervenciones que fomenten un buen clima escolar mediante buenas 
relaciones interpersonales, comunicación asertiva, empatía y respeto mutuo, entre otras (Bright et al., 2020; Calvete et al., 2019; Cowie y Olafsson, 2000; Diamond et al., 2019; Dillon y Lochman, 2019; Díaz-Aguado, 2005; Merrell et al., 2008; Moore et al., 2019; Nieh y Wu, 2018; Pertegal et al., 2010; Rapee et al., 2020; Salimi et al., 2019; Van y Roseth, 2019).

Existen diferentes estrategias de intervención dirigidas al cambio de la organización escolar, la formación de docentes y los programas específicos sobre situaciones y fenómenos de violencia en niños y adolescentes víctimas o en riesgo. Las estrategias centradas en la organización escolar parten de la idea de que el contexto educativo es un sistema general de convivencia que hay que gobernar y dinamizar de forma que facilite las relaciones positivas y prevenga conductas negativas por medio del diálogo, la cooperación y el enriquecimiento mutuo entre cada uno de los miembros (Del Rey y Ortega, 2001).

Dentro de los programas de intervención enfocados en la formación de docentes, es importante resaltar que el propósito es fortalecer las relaciones profesionales de los educandos mediante el trabajo en equipo y cursos de educación para la convivencia, resolución de conflictos, habilidades sociales y prevención de la violencia (Del Rey y Ortega, 2001).

Por otro lado, dentro de las propuestas de actividades para desarrollar en el aula de clase, se crean programas de educación en valores, clima social, cooperativismo, emociones, sentimientos y diálogos orientados a disminuir y prevenir problemas de convivencia y clima escolar. De igual manera, se busca el respeto mutuo y la solidaridad mediante de actividades de reflexión a través de situaciones vividas que hayan ocasionado consecuencias debido a conductas antisociales o violentas dentro del ámbito educativo (Del Rey y Ortega, 2001).

\section{ACOSO ESCOLAR}

El acoso escolar, también denominado bullying, es un tipo específico de violencia entre compañeros, caracterizado por comportamientos violentos como maltrato físico y psicológico con la intencionalidad de hacer daño de manera frecuente a la misma persona (Leganés, 2013). Se ha convertido en un problema mundial en los diferentes contextos educativos debido a las afectaciones que puede ocasionar en víctimas, agresores e incluso espectadores (Castro, Niño y Fernández, 2020). Este tipo de violencia entre compañeros se presenta principalmente en el entorno escolar; generalmente, tanto las víctimas como los agresores pertenecen al mismo grado académico y no cuentan en su mayoría con la supervisión constante de un docente que evite ese tipo de acciones y conductas (Resett, 2016).

\section{Tipos de acoso escolar}

Según Enríquez y Garzón (2015), el acoso escolar se manifiesta de diferentes formas:

- Acoso físico: hace referencia al daño o riesgo que ocasiona un grupo de personas sobre la integridad física de la víctima mediante conductas agresivas como patadas, empujones, pellizcos, golpes o palizas.

- Acoso verbal: hace referencia a las amenazas verbales que recibe la víctima por parte de sus compañeros a través de burlas y humillaciones constantes relacionadas con su aspecto físico, religión, género, discapacidad y orientación sexual.

- Acoso relacional o social: hace referencia al rechazo social y la difusión de rumores maliciosos por parte de sus compañeros debido a su aspecto físico, forma de actuar y pensar.

- Acoso virtual (cyber-bullying): se relaciona con las diferentes formas de acoso u hosti- 
gamiento entre iguales a través de espacios virtuales (internet, celular) y de manera anónima para que la víctima desconozca quién es el agresor.

\section{Características de los actores del acoso escolar}

\section{AGRESORES}

Dentro de las características de los estudiantes que practican conductas de acoso escolar hacia sus compañeros, se evidencia un alto grado de impulsividad e incapacidad de autocontrol a las normas y reglas establecidas, son extrovertidos, con baja resistencia a la frustración y sentimientos de autosuficiencia. Los participantes de género masculino se caracterizan por ejercer conductas de violencia física a las víctimas de forma grupal, mientras que las niñas practican intimidaciones de tipo psicológico, emocional y afectivo a nivel individual (Santoyo y Frías, 2014).

\section{VÍCTIMAS}

- Víctimas pasivas: se caracterizan principalmente por no responder a los ataques por parte de sus compañeros, presentan rasgos de inseguridad, ansiedad, baja autoestima y dependencia. Los individuos víctimas de la violencia escolar suelen ser retraídos, temerosos y con tendencia a presentar problemas emocionales.

- Víctimas provocativas: se caracterizan por presentar conductas violentas y desafiantes, adoptando el rol de agresor y compartiendo todas sus características.

Las víctimas del acoso escolar enfrentan diferentes situaciones negativas dentro del contexto educativo, lo que ocasiona pérdida de motivación por ir a la escuela. Por lo tanto, la víctima debe enfrentarse a sus victimarios y a la insistencia de los progenitores de no abandonar la escuela, la que se convierte en un espacio de riesgo, temor e inseguridad (Santoyo y Frías, 2014).

\section{ESPECTADORES}

Los espectadores o testigos son aquellos individuos que observan la agresión desde afuera, evitan dar información respecto a las situaciones que evidencian sobre maltrato físico o psicológico por parte de sus compañeros con la víctima. Prefieren guardar silencio por miedo a las consecuencias que esto les pueda ocasionar (golpes, rechazo o convertirse en víctimas; Santoyo y Frías, 2014).

\section{Consecuencias del acoso escolar}

La prevalencia del acoso escolar en las escuelas de diferentes países varía desde menos del 10\% hasta más del 65\% entre niños y adolescentes (Organización de las Naciones Unidas para la Educación, la Ciencia y la Cultura [Unesco], 2017). Estas experiencias de victimización por bullying han generado problemas de salud mental en cada uno de los autores (víctima, acosador y espectador), lo que limita un buen desarrollo psicológico, un adecuado aprendizaje escolar y la inclusión en la sociedad (Carney y Merrel, 2001; Griffin y Gross, 2004).

El acoso escolar provoca efectos negativos en toda la comunidad educativa, afecta las relaciones interpersonales y el buen clima escolar. Para la víctima, este tipo de violencia escolar ocasiona efectos negativos a nivel emocional y psicológico como síntomas depresivos, ansiedad, baja autoestima, cambios en los patrones de sueño, pérdida de interés en las actividades diarias, fracaso escolar, sentimientos de tristeza y soledad, lo que va acompañado de somatizaciones como dolor de cabeza, dolor de estómago, náuseas, vómitos, pesadillas nocturnas y no querer volver a la escuela, entre otros 
(Bonilla et al., 2017; Brendgen y Poulin, 2018; Lugones y Ramírez, 2017; Polo et al., 2014). Asimismo, es importante destacar que los jóvenes que sufren acoso escolar tienden a presentar falencias en el uso de estrategias para la resolución de conflictos, quedan expuestos a este tipo de problemáticas y encuentran como solución enfrentarla, resistir o escapar de ella.

Por otro lado, cabe mencionar que los perpetradores tienen mayor prevalencia de adquirir una personalidad antisocial, consumir sustancias psicoactivas y padecer trastornos ansioso-depresivos (Albores-Gallo et al., 2011; Cañas-Pardo, 2017). La mayoría de estos actores tienen dificultades de adaptación y emplean un comportamiento agresivo como la única manera de comunicarse y ser aceptados socialmente por un grupo determinado de compañeros. Del mismo modo, los espectadores tienden a presentar sentimientos de culpabilidad al no poder interceder en las situaciones de maltrato y hostigamiento por parte de sus compañeros con la víctima. No obstante, este tipo de violencia afecta también a los estudiantes no involucrados en su aprendizaje y rendimiento académico.

\section{Factores de riesgo}

No existe un factor único que ponga a un niño en riesgo de ser acosado o acosar a otros. El acoso puede ocurrir en cualquier lugar, en la ciudad o en áreas rurales, así como en la escuela, el colegio e incluso en la universidad. Sin embargo, existen múltiples elementos en el ámbito familiar, individual y social que incrementan el riesgo de ser acosados o convertirse en acosadores. A su vez, algunos procesos de interacción social movilizan la difusión de información falsa y dañina que puede conducir a un aumento de las tasas de acoso contra ciertos grupos de personas. Algunos factores de riesgo asociados al desarrollo de conductas de acoso se exponen en la tabla 1.

\section{TABLA 1}

Factores de riesgo para el desarrollo de conductas de acoso

\begin{tabular}{|c|c|}
\hline Ámbito & Factores de riesgo asociados a la conducta de acoso \\
\hline \multirow{4}{*}{ Familiar } & Ausencia de pautas de crianza \\
\hline & Normalización del comportamiento agresivo \\
\hline & Ausencia o escasez de supervisión \\
\hline & Mala comunicación (padre-hijo) \\
\hline \multirow{5}{*}{ Individual } & Violencia en el hogar \\
\hline & Violencia de género \\
\hline & Problemas de ansiedad, depresión \\
\hline & Dificultades para relacionarse \\
\hline & Inestabilidad emocional \\
\hline \multirow{2}{*}{ Social } & Exposición continua a la violencia en medios de comunicación \\
\hline & Contexto escolar que no promueve relaciones positivas \\
\hline
\end{tabular}

Nota: elaboración propia. 


\section{Intervención en el acoso escolar}

La prevención del acoso escolar es un elemento clave para mejorar la salud mental y el bienestar psicológico de los niños y adolescentes. Por esta razón, surge la necesidad de prestar especial atención a los niños víctimas del bullying, ya que corren el riesgo de desarrollar problemas psicológicos y emocionales. Asimismo, están más vulnerables a padecer trastornos psiquiátricos en la edad adulta temprana (Sourander et al., 2007).

Algunas investigaciones demuestran que la intervención temprana es un elemento clave tanto para la promoción del comportamiento positivo como para la prevención de la violencia y sus consecuencias, particularmente en los individuos con mayor desventaja social (Fernández y Vivar, 2010; Hidalgo, 2016).

Por lo tanto, muchos estudios se han enfocado en el desarrollo de programas de intervención para la prevención y reducción del acoso escolar en los diferentes contextos educativos, a través de componentes educativos relacionados con la mejora de las habilidades emocionales, espacios de comunicación asertiva, habilidades de toma de decisiones, estrategias de afrontamiento ante situaciones estresantes y resolución de conflictos (Bright et al., 2020; Calvete et al., 2019; Cowie y Olafsson, 2000; Diamond et al., 2019). Este tipo de intervenciones y programas educativos se han desarrollado para abordar los diferentes factores de riesgo asociados con el bullying, ya que los entornos escolares permiten la construcción de relaciones que fortalecen la convivencia.

\section{Neuropsicología del acoso escolar}

El abuso, el maltrato y la privación durante los primeros años de vida obstaculizan el desarrollo normal del cerebro. Variables como la edad, la duración y la intensidad pueden ocasionar alteraciones en ciertas estructuras cerebrales, principalmente en la reducción del cuerpo calloso y volumen del cerebelo (Amores y Mateos, 2017). Durante edades tempranas el infante necesita afecto, estimulación e interacción con el entorno para el adecuado desarrollo de las áreas cerebrales que regulan la socialización (Fernández-Daza, 2016). Por lo tanto, el apoyo emocional y refuerzo positivo son aspectos que se asocian con estilos de crianza que favorecen las habilidades sociales y los vínculos afectivos.

Del mismo modo, algunas investigaciones han mostrado evidencia de que los déficits en el funcionamiento conductual y cognitivo de los niños que han sido víctimas de privaciones severas (maltratos o abusos de manera frecuente) aumentan los niveles de cortisol y pueden estar relacionados con alteraciones estructurales y funcionales del cerebro, específicamente con una disfunción del eje hipotálamo-pituitaria-adrenal e hipoactividad en varias regiones del cerebro, incluyendo regiones de la corteza prefrontal, sistema límbico y paralímbico (Fernández-Daza, 2016). Asimismo, se encuentran vulnerables a padecer trastornos de salud mental.

En otras palabras, la experiencia social en edades tempranas tiene un papel fundamental en la organización de circuitos neuronales que se vinculan con el autocontrol y la interacción social. La discriminación, la estigmatización social y los niveles altos de estrés pueden desencadenar en los infantes dificultades para establecer relaciones de apego seguros y buenas relaciones interpersonales (Fernández-Daza, 2016).

Por lo tanto, el bullying ha sido relacionado con emociones como el miedo; a nivel de estructuras cerebrales, se ha indicado que cuando el estímulo llega a la amígdala, se reconoce y se asocia con la dimensión afectiva correspondiente. La amígdala simboliza el sistema central del miedo y es esencial en la adquisición 
y expresión del miedo condicionado, así como de la ansiedad (Fernández-Daza, 2016).

En específico, el área hipotalámica lateral tiene un papel importante en la respuesta cardiovascular a estímulos de miedo (Fernández-Daza, 2016). Las señales llegan a la zona lateral del hipotálamo desde la amígdala y otras áreas. El hipocampo es la estructura cerebral responsable del condicionamiento al miedo, lo que indica que los estados emocionales provocados por ese tipo de violencia escolar como lo es el bullying generan desencadenantes negativos a nivel de conexiones y redes neuronales que podrían alterar la arquitectura cerebral y su actividad, sobre la base de sustratos neurales del sistema defensivo y de las emociones negativas implicadas (miedo, ansiedad, tristeza, depresión).

En conclusión, estudios han demostrado que los acontecimientos vitales de estrés prolongado relacionados con experiencias de violencia escolar - principalmente el acoso escolar - se asocian con síntomas internalizados como la depresión, la ansiedad y el trauma.

\section{COGNICIÓN SOCIAL}

La cognición social hace referencia a cualquier proceso cognitivo relacionado con la capacidad de comprenderse a sí mismo y comprender a los demás como individuos dentro de un contexto social; incluye las maneras en las que podemos descifrar la emoción en el rostro de una persona y deducir las intenciones del otro. Este proceso tiene un correlato neurobiológico que permite que tanto los humanos como los animales interpreten adecuadamente los signos sociales, lo cual se considera un eje fundamental en el éxito de la conducta social. Este proceso empieza primero con la percepción de los rostros, los cuerpos y las acciones de los demás. Durante las primeras experiencias escolares del infante, las inferencias empáticas o no empáticas pueden carecer por completo de afectos asociados; sin embargo, a medida que van creciendo, se vuelven un poco más precisos frente a los sentimientos propios y de los demás, lo que indica que los procesos cognitivos se van desarrollando con el tiempo en la medida en que el niño adquiere experiencias en el contexto social (Betancourt et al., 2014).

La cognición social y la empatía hacen uso de las emociones para deducir la forma correcta de actuar frente a una situación específica. Por esta razón, existen diferentes sustratos neurológicos que se comprometen al poder dar una respuesta adecuada; especialmente, se ha relacionado la corteza prefrontal medial como un área que integra la información que se obtiene de los procesos atencionales y que permite la manifestación de funciones ejecutivas como la planeación y la toma de decisiones.

Se han propuesto diversas áreas cerebrales implicadas; a nivel implícito se involucran el área fusiforme, el giro frontal inferior y las áreas premotoras, mientras que a nivel explícito se encuentra la corteza prefrontal medial y el área temporoparietal.

En la percepción de las emociones predominan las expresiones faciales y la expresión de los ojos; algunos autores plantean que, al observar un estado afectivo emocional en otra persona, se activa un sistema neuronal que dispara las mismas regiones cerebrales, lo que explica no solo la capacidad de respuesta ante las necesidades, sino el proceso de empatía entre individuos (Betancourt et al., 2014). 


\section{ACTIVIDADES}

\section{Actividad 1}

Aplicación: grupal.

Objetivo: reconocer emociones a través de diferentes sucesos de la vida diaria.

Duración: media hora.

Instrucciones: vamos a estar muy atentos porque a continuación vamos a presentarles, en una pantalla, fotografías de personajes que expresan emociones diferentes (alegría, tristeza, miedo, asco, enojo, sorpresa). Cada niño deberá estar muy atento porque seguidamente se contará una historia con el fin de que identifiquen qué emoción les generaría estar en dicha situación.

\section{HISTORIA 1}

Camila es una niña de ocho años a quien no le gusta ir a la escuela porque todos sus compañeritos la molestan por ser gorda; la mayoría del tiempo está expuesta a que la rechacen y le pongan apodos desagradables debido a su aspecto físico.

Emoción correspondiente: tristeza.

- ¿Creen que los compañeros de Camila están actuando mal?

- ¿Cuál sería la forma correcta de actuar con Camila?

- ¿Consideran que debemos aceptarnos y respetarnos unos a otros?

\section{HISTORIA 2}

Felipe es un niño de bajos recursos económicos que sobresale en el aula de clase por su inteligencia y compañerismo. Frecuentemente recibe halagos por parte de sus compañeros y docentes. Al finalizar el año, le hicieron un gran reconocimiento.

Emoción correspondiente: alegría.

\section{HISTORIA 3}

Andrés iba de camino hacia el parque a pasear a su mascota, decidió soltarla para que jugara, cuando de repente un señor se acercó y le dio una patada. Andrés sintió impotencia por no poder defenderla.

Emoción correspondiente: enojo.

\section{HISTORIA 4}

Samuel fue a casa de sus abuelos y descubrió que le tenían una mascota de regalo por el día de sus cumpleaños.

Emoción correspondiente: sorpresa.

\section{HISTORIA 5}

Sofía iba de paseo en compañía de su hermano mayor, se cruzaron con una serpiente muy grande y decidieron huir. Al llegar a casa, sus padres notaron que venían muy agitados y decidieron preguntar por lo sucedido.

Emoción correspondiente: miedo.

\section{HISTORIA 6}

Santiago llegó en compañía de su mejor amigo a un restaurante muy reconocido de la ciudad, en medio de las charlas miró hacia la cocina y vio que había varios ratones alrededor.

Emoción correspondiente: asco. 


\section{Actividad 2}

Aplicación: grupal.

Objetivo: sensibilizar sobre el daño que pueden ocasionar las palabras negativas.

Duración: una hora.

Instrucciones: la actividad consiste en leerles a los alumnos una cantidad de palabras que se encuentran en el tablero, con el fin de que inicialmente seleccionen solo aquellas frases que les gustaría escuchar por parte de sus compañeros y seguidamente aquellas frases que no les gustaría recibir porque consideran que son ofensivas y los hacen sentir mal. Después, se les dará el espacio a algunos alumnos para que cuenten una situación específica en la que se hayan sentido mal y una en la que se hayan sentido bien.
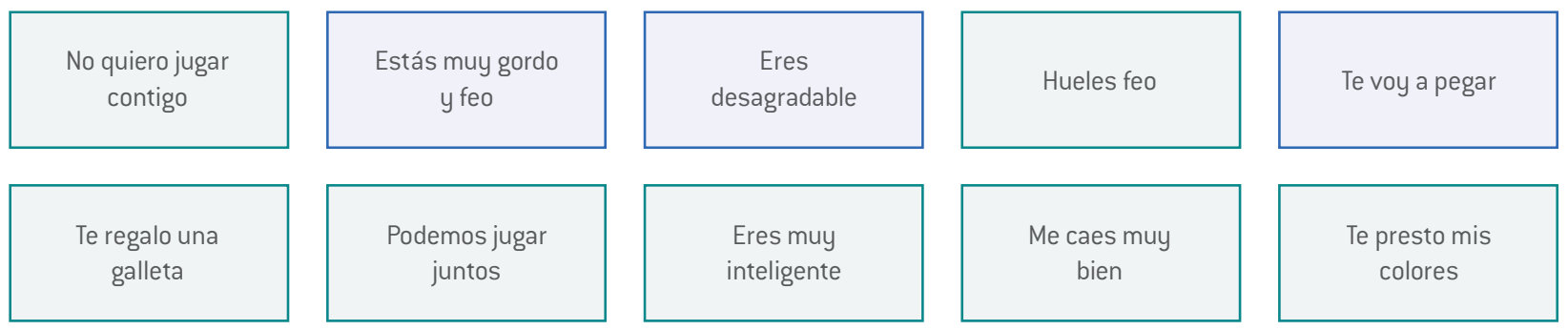

Te presto mis colores

Finalmente se resuelven una serie de preguntas:

- ¿Cuál es la forma correcta de actuar con los compañeros?

- ¿Las malas palabras hacen sentir mal a nuestros compañeros?

- ¿Consideran que cuando nos dicen palabras agradables nos sentimos felices?

\section{Actividad 3}

Aplicación: grupal.

Objetivo: identificar los perfiles de los actores del bullying (acoso escolar).

Duración: una hora.

Instrucciones: la actividad consiste inicialmente en darles a conocer a los alumnos, mediante tres historias, cada una de las características que se asocian a los perfiles de los actores del bullying (acoso escolar).

\section{HISTORIA 1}

Aurelio es un niño muy tímido a quien se le dificulta relacionarse con los demás, todos sus compañeritos se le burlan frecuentemente porque tiene una discapacidad visual. Cada vez que llega a clase, le ponen apodos y en ocasiones han intentado agredirlo físicamente.

Rol: víctima.

\section{HISTORIA 2}

Bruno es un niño que se caracteriza por ser agresivo con sus compañeros de clase, frecuentemente persigue a su compañero Camilo para agredirlo verbalmente y lastimarlo hasta hacerlo llorar.

Rol: agresivo.

\section{HISTORIA 3}

Camila observa todas las mañanas en el aula de clase que unas estudiantes de grado superior empujan y agreden verbalmente a su 
mejor amiga. Cada vez que observa dicha situación, prefiere huir y no comentarle a nadie.

Rol: pasivo.

Finalizado el ejercicio anterior, se les presentará a cada uno de los estudiantes una ruleta con situaciones que representan características de los actores del bullying (víctima, agresor y espectador; figura 1). La finalidad de la actividad es que cada uno de los participantes identifique cada una de las situaciones correspondientes y las categorice en víctima, agresor o espectador.

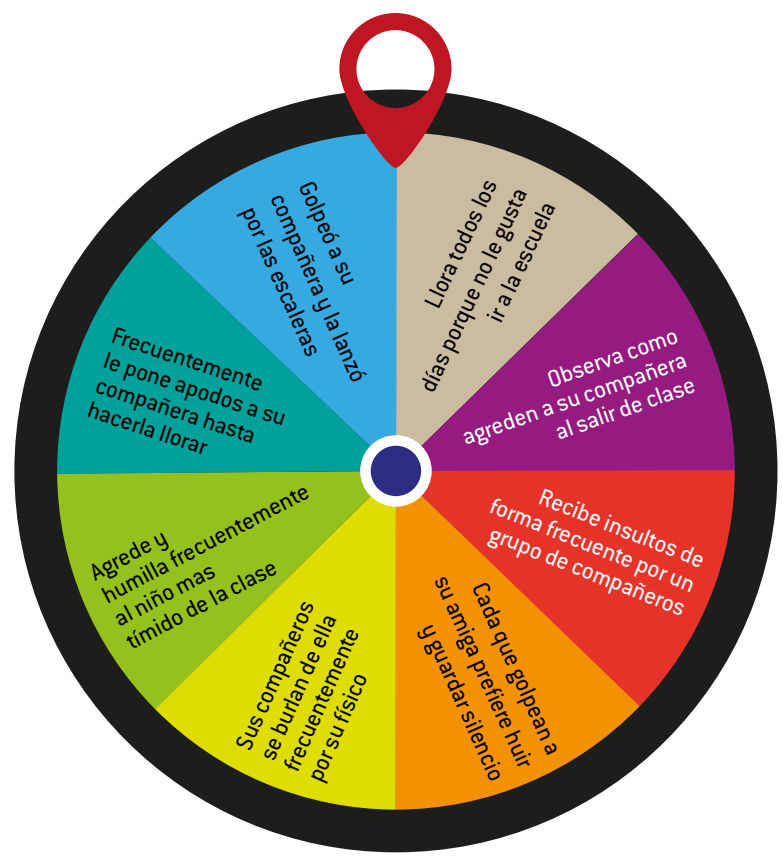

FIGURA 1. Juego de la ruleta. Elaboración propia.

\section{Actividad 4}

Aplicación: grupal.

Objetivo: hacer que cada uno de los participantes identifiquen situaciones que pueden ocasionar violencia escolar y cuál es la forma correcta de actuar frente a estas situaciones.

Duración: 1 hora.

Instrucciones: realizar juego de roles a través de grupos que permitan representar una historia sobre el bullying y reconocimiento de emociones; se facilitará a cada uno de los grupos unas figuras (conejo, cuadrado, triangulo), con el propósito de que puedan representar la situación seleccionada (figura 2).

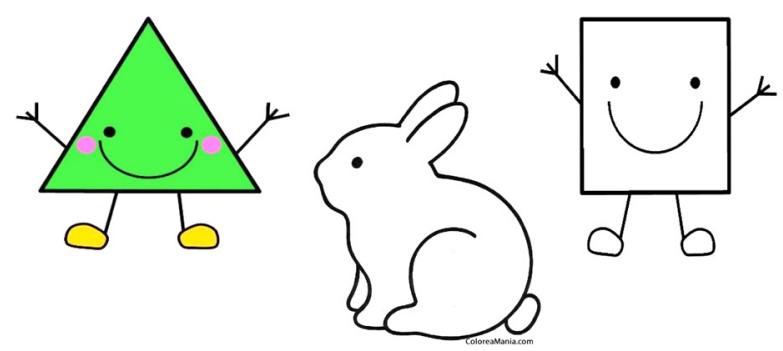

FIGURA 2. Formas del acoso escolar. Tomadas de Pinterest: https://bit.ly/3srJsnV, https://bit.ly/3B49JfT, https://bit. ly/3GDOqDO

\section{Actividad 5}

Aplicación: individual.

Objetivo: identificar cuáles son las emociones son consideradas como agradables y desagradables para cada uno de los niños participantes.

Duración: una hora.

Instrucciones: inicialmente se les mostrará a los niños un cartel con las emociones (alegría, tristeza, enojo, asco, rabia, miedo; figura 3); seguidamente se facilitará a cada uno de los niños una hoja en blanco con el propósito de que dibujen la emoción que consideran más agradable y desagradable en su vida cotidiana y expliquen por qué motivo escogieron dichas emociones.

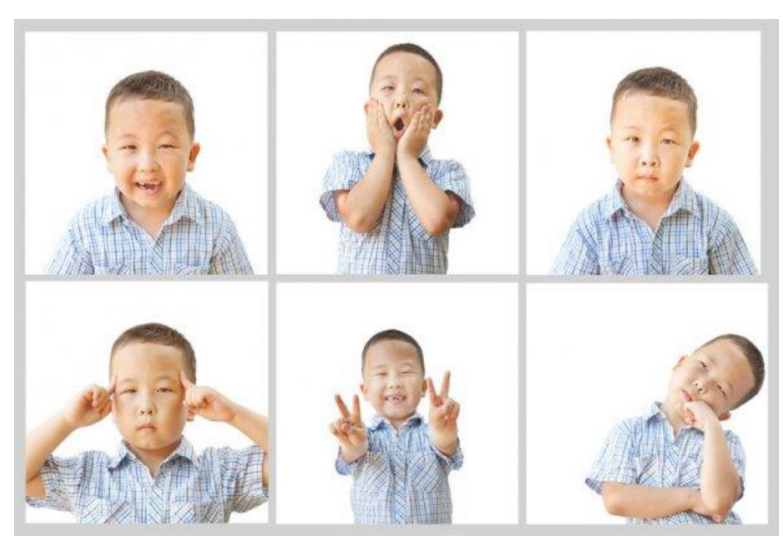

FIGURA 3. Reconocimiento de emociones. Tomada de https://bit.ly/3LhKpYE 


\section{Actividad 6}

Aplicación: individual.

Objetivo: identificar las emociones que se pueden generar a través de diferentes melodías.

Duración: una hora.

Instrucciones: inicialmente se pondrá a cada uno de los participantes a escuchar melodías que representen diferentes emociones; seguidamente, se les facilitará un papelito con el propósito de que logren escribir la emoción seleccionada de acuerdo con los audios presentados anteriormente.

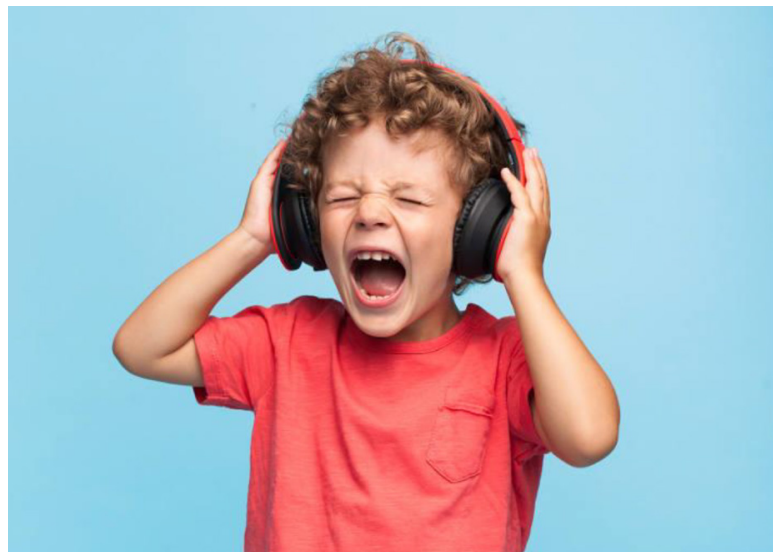

FIGURA 4. Identificar mi estado de ánimo. Tomada de https://bit.ly/35YoJR1

\section{Actividad 7}

Aplicación: grupal.

Objetivo: lograr que los participantes identifiquen mediante mímicas los roles que se evidencian a través de situaciones que representan el bullying en los diferentes contextos que frecuentan los niños.

Duración: una hora.

Instrucciones: la actividad consiste en escoger dos niños del grupo de forma voluntaria; se les mostrará una situación de acoso con el fin de que logren interpretarla de forma gestual y puedan representarla frente a sus compañeros de clase; seguidamente, el resto del grupo deberá adivinar quién es el acosador y quién la víctima. Durante el desarrollo de la actividad, el juego de roles se llevará a cabo entre los diferentes participantes del grupo.

\section{Actividad 8}

Aplicación: grupal.

Objetivo: crear lazos de amistad a través de espacios de comunicación.

Duración: una hora.

Instrucciones: se pide al grupo de niños participantes que realicen una mesa redonda; se les harán preguntas personales al azar tales como las siguientes:

- ¿Cuál es tu color favorito?

- ¿Cuál es tu película favorita?

- ¿Con quién vives?

- ¿Cuál es tu comida favorita?

- ¿Tienes mascotas? ¿Cómo se llama(n)?

- ¿Cuántos años tienes?

Finalmente, después de que cada uno de los niños participa en la actividad, se procederá a preguntar al azar a cualquiera de ellos cuál fue la respuesta de su compañero con el propósito de generar empatía y comunicación.

\section{Actividad 9}

Aplicación: grupal.

Objetivo: aprender a identificar palabras que se asocian al acoso escolar a través de una sopa de letras.

Duración: media hora.

Instrucciones: como primera medida, se le pide a cada uno de los niños participantes que formen parejas; seguidamente, se le facilita a cada uno de los grupos una hoja que contiene una sopa de letras (figura 4), con el fin de que encuentren las palabras que están relacionadas con el acoso escolar. 


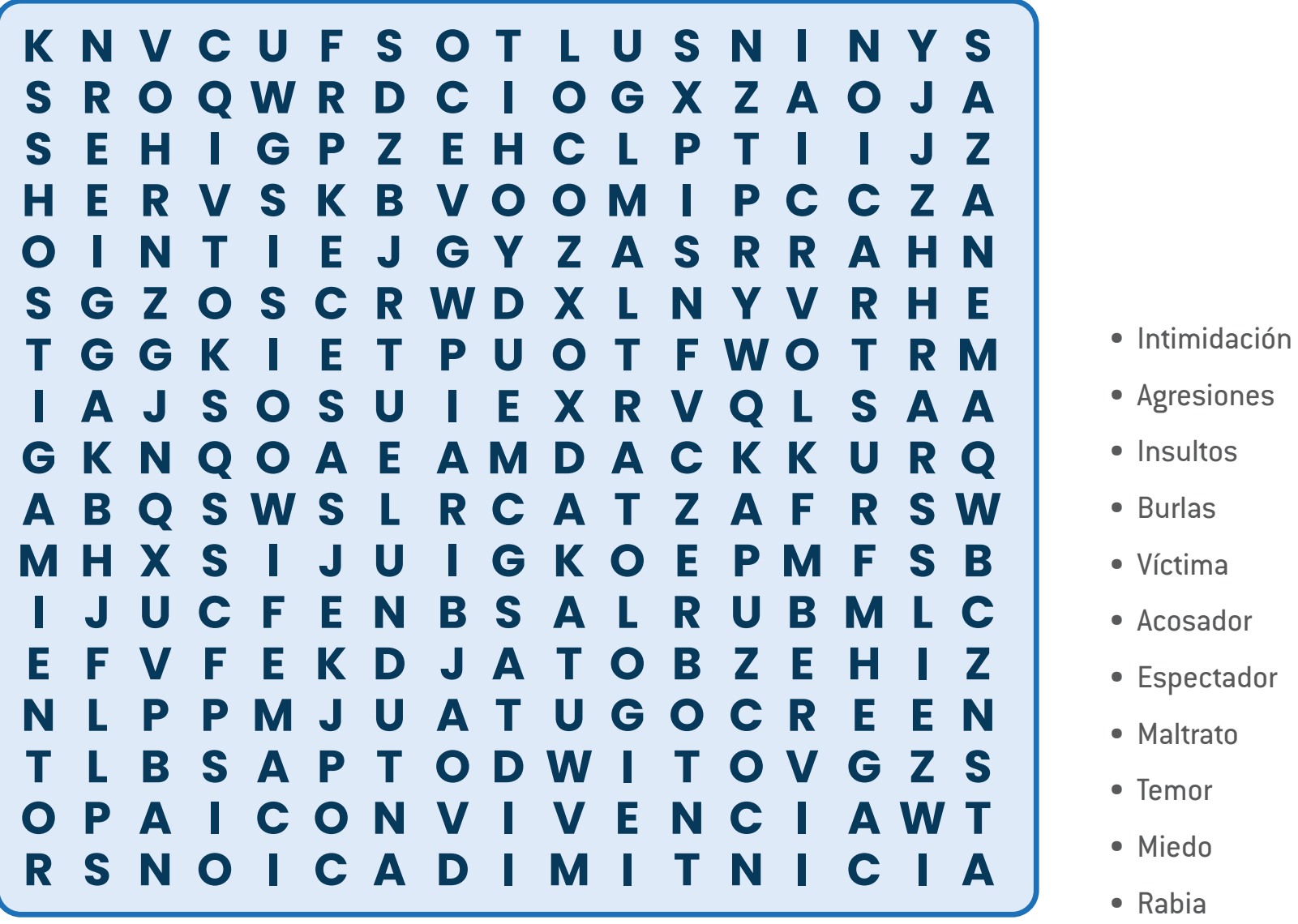

FIGURA 4. Juego de sopa de letras. Elaboración propia.

\section{Actividad 10}

Aplicación: grupal.

Objetivo: aprender a identificar el concepto del bullying y sus consecuencias.

Duración: una hora.

Instrucciones: inicialmente se les mostrará a los niños un video sobre el bullying y sus consecuencias; seguidamente, se entregará a cada uno de los estudiantes una hoja con diferentes preguntas de verdadero o falso sobre la temática de acoso escolar, con el fin de que encierren en un círculo la respuesta que consideren correcta de acuerdo con lo aprendido en el video presentado inicialmente. 


\section{REFERENCIAS}

Albores-Gallo, L., Sauceda-García, J., Ruiz-Velasco, S. y Roque-Santiago, E. (2011). El acoso escolar (bullying) y su asociación con trastornos psiquiátricos en una muestra de escolares en México. Salud Pública de México, 53(3), 220-227. https://www.medigraphic.com/cgi-bin/new/resumen.cgi?IDARTICULO=30158

Amores, A. y Mateos, R. (2017). Revisión de la neuropsicología del maltrato infantil: la neuropsicología y el perfil neuropsicológico de las víctimas de abusos en la infancia. Psicología Educativa, 23(2), 81-88. https:// www.redalyc.org/articulo.oa?id=613765228002

Ayala-Carrillo, M. R. (2015). Violencia escolar: un problema complejo. Ra Ximhai, 11(4), 493-509. https://www. redalyc.org/pdf/461/46142596036.pdf

Betancourt, Y. U., Restrepo, J. G., Pinzón, S., Acosta, J., Diaz, M. y Bonilla, C. (2014). Vínculo afectivo en pares y cognición social en la infancia intermedia. International Journal of Psychological Research, 7(2), 51-63. https:// www.redalyc.org/articulo.oa?id=299032684006

Bonilla, J., González, A. y Bonilla, G. (2017). Características neuroendocrinas del trauma temprano y su relación con el bullying. Revista Ecuatoriana de Neurología, 26(3), 258-265. https://pesquisa.bvsalud.org/portal/ resource/pt/biblio-1003991

Brendgen, M. y Poulin, F. (2018). Continued Bullying Victimization from Childhood to Young Adulthood: A Longitudinal Study of Mediating and Protective Factors. Journal of Abnormal Child Psychology, 46(1), 27-39. https://pubmed.ncbi.nlm.nih.gov/28608169/

Bright, M. A., Sayedul Huq, M., Patel, S., Miller, M. D. y Finkelhor, D. (2020). Child Safety Matters: Randomized Control Trial of a School-Based, Child Victimization Prevention Curriculum. Journal of Interpersonal Violence, 37(1-2), 538-556. https://doi.org/10.1177/0886260520909185

Calvete, E., Orue, I., Fernández-González, L. y Prieto-Fidalgo, A. (2019). Effects of an Incremental Theory of Personality Intervention on the Reciprocity between Bullying and Cyberbullying Victimization and Perpetration in Adolescents. PLoS one, 14(11). https://doi.org/10.1371/journal.pone.0224755

Cañas-Pardo, E. (2017). Acoso escolar: características, factores de riesgo y consecuencias. Revista Doctorado Uмн, 3(1), 7-17. https://revistas.innovacionumh.es/index.php/doctorado/article/view/635

Carney. A. G. y Merrell, K. W. (2001). Bullying in Schools: Perspectives on Understanding and Preventing an International Problem. School Psychology International, 22(3), 364-382. https://psycnet.apa.org/record/2001-11008-010

Castro-Robles, A., Niño-Vega, J. y Fernández-Morales, F. (2020). El acoso escolar como oportunidad para mejorar la convivencia en el aula. Educación y Humanismo, 22(38). https://doi.org/10.17081/eduhum.22.38.3675

Cowie, H. y Olafsson, R. (2000). The Role of Peer Support in Helping the Victims of Bullying in a School with High Levels of Aggression. School Psychology International, 21(1), 79-95. https://doi.org/10.1177/0143034300211006

Del Rey, R. y Ortega, R. (2001). Programas para la prevención de la violencia escolar en España: la respuesta de las comunidades autónomas. Revista Interuniversitaria de Formación del Profesorado, 41, 133-145. https:// www.redalyc.org/pdf/274/27404109.pdf

Diamond, A., Lee, C., Senften, P., Lam, A. y Abbott, D. (2019). Randomized Control Trial of Tools of the Mind: Marked Benefits to Kindergarten Children and their Teachers. PLoS One, 14(9). https://doi.org/10.1371/ journal.pone.0222447 
Díaz-Aguado Jalón, M. J. (2005). Por qué se produce la violencia escolar y cómo prevenirla. Revista Iberoamericana de Educación, 37. https://doi.org/10.35362/rie370838

Dillon, C. E. y Lochman, J. E. (2019). Correcting for norm misperception of anti-bullying attitudes. International Journal of Behavioral Development, 9, 1-10. https://doi.org/10.1177/0165025419860598

Enríquez, M. F.y Garzón, F. (2015). El acoso escolar. Saber, Ciencia y Libertad, 10(1). http://dx.doi.org/10.18041/23823240/saber.2015v10n1.983

Fernández, M. J. M. y Vivar, D. M. (2010). La escuela inclusiva ante el acoso escolar. Estrategias de prevención para el profesorado. Revista de Educación Inclusiva, 3(3), 123-136. https://revistaeducacioninclusiva.es/index.php/REI/article/view/177

Fernández-Daza, M. (2016). Neuropsicología del acoso escolar: función mediadora de la conducta prosocial. Revista Mexicana de Neurociencia, 17(6), 106-119. https://www.medigraphic.com/cgi-bin/new/resumen. cgi?IDARTICULO=69356

Hidalgo-Ripodas, L. (2016). Previniendo el acoso escolar desde la etapa de Educación Infantil [Tesis de grado]. Universidad Internacional de La Rioja.

Leganés, E. (2013). Una propuesta de intervención para prevenir el acoso escolar desde una perspectiva sociogrupal. Psychology, Society \& Education, 5(1), 21-40. https://dialnet.unirioja.es/servlet/articulo?codigo $=4489368$

Lugones Botell, M. y Ramírez Bermúdez, M. (2017). Bullying: aspectos históricos, culturales y sus consecuencias para la salud. Revista Cubana de Medicina General Integral, 33(1), 154-162. https://pesquisa.bvsalud.org/ portal/resource/pt/biblio-901155

Martínez, Á. C. (2010). Pautas de crianza y desarrollo socioafectivo en la infancia. Diversitas, 6(1), 111-121. https://www.redalyc.org/pdf/679/67916261009.pdf

Merrell, K. W., Gueldner, B. A., Ross, S. W. e Isava, D. M. (2008). How Effective Are School Bullying Intervention Programs? A Meta-Analysis of Intervention Research. School Psychology Quarterly, 23, 26-42. https://psycnet.apa.org/record/2008-04403-004

Moore, B., Woodcock, S. y Dudley, D. (2019). Developing Wellbeing through a Randomized Controlled Trial of a Martial Arts-Based Intervention: An Alternative to the Anti-Bullying Approach. International Journal of Environmental Research and Public Health, 16(1), 81. https://pubmed.ncbi.nlm.nih.gov/30597946/

Nieh, H. P. y Wu, W. C. (2018). Effects of a Collaborative Board Game on Bullying Intervention: A Group-Randomized Controlled Trial. Journal of School Health, 88(10), 725-733. https://pubmed.ncbi.nlm.nih.gov/30203481/

Pertegal, M. Á., Oliva, A. y Hernando, Á. (2010). Los programas escolares como promotores del desarrollo positivo adolescente. Cultura y Educación, 22(1), 53-66. https://personal.us.es/oliva/04.\%20PERTEGAL.pdf

Polo, I., León, B, Fajardo, F., Felipe, E. y Palacios, V. (2014). Perfiles de personalidad en víctimas de acoso escolar. INFAD Revista de Psicología, 1(1), 409-416. https://www.redalyc.org/pdf/3498/349851780042.pdf

Prieto, G. (2005). Violencia escolar y vida cotidiana en la escuela secundaria. Revista Mexicana de Investigación Educativa, 10(27), 1005-1026. https://www.redalyc.org/pdf/140/14002703.pdf

Rapee, R. M., Shaw, T., Hunt, C., Bussey, K., Hudson, J. L., Mihalopoulos, C. y Cordin, T. (2020). Combining WholeSchool and Targeted Programs for the Reduction of Bullying Victimization: A Randomized, Effectiveness Trial. Aggressive Behavior, 46(3), 193-209. https://onlinelibrary.wiley.com/doi/10.1002/ab.21881 
Resett, S. A. (2016). Una caracterización del acoso escolar en la adolescencia: quiénes, cómo y dónde se agrede. Perspectivas en Psicología, 13(1), 11-20. https://ri.conicet.gov.ar/handle/11336/106484

Rodríguez, Y. R., Gamboa, G. R. y Hernández, D. S. (2020). Características de la violencia escolar en adolescentes del municipio Camagüey. EduSol, 20(71), 158-169. https://www.redalyc.org/journal/4757/475764265012/html/

Salimi, N., Karimi-Shahanjarini, A., Rezapur-Shahkolai, F., Hamzeh, B., Roshanaei, G. y Babamiri, M. (2019). The Effect of an Anti-Bullying Intervention on Male Students' Bullying-Victimization Behaviors and Social Competence: A Randomized Controlled Trial in Deprived Urban Areas. Journal of Research in Health Sciences, 19(4). https://pubmed.ncbi.nlm.nih.gov/32291360/

Santoyo D. y Frías, S. M. (2014). Acoso escolar en México: actores involucrados y sus Características. Revista Latinoamericana de Estudios Educativos, 44(4), 13-41. https://www.redalyc.org/pdf/270/27032872002.pdf

Sourander, A., Jensen, P., Rönning, J. A., Niemelä, S., Helenius, H., Sillanmäki, L. y Almqvist, F. (2007). What is the Early Adulthood Outcome of Boys who Bully or Are Bullied in Childhood? The Finnish "From a Boy to a Man" Study. Pediatrics, 120(2), 397-404.

Organización de las Naciones Unidas para la Educación, la Ciencia y la Cultura. (Unesco). (2017). School Violence and Bullying: Global Status Report. Unesco.

Van Ryzin, M. J. y Roseth, C. J. (2019). Effects of Cooperative Learning on Peer Relations, Empathy, and Bullying in Middle School. Aggressive Behavior, 45(6), 643-651. https://www.ncbi.nlm.nih.gov/pmc/articles/ PMC7413077/ 


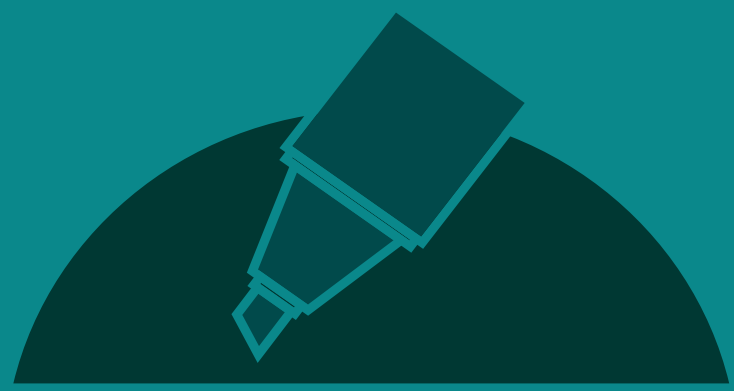

Guías

prácticas 